\title{
An Update Review on Listeria Infection in Pregnancy
}

\author{
Zhaoyun Wang ${ }^{1, *}$ \\ Xiaojing Tao $\mathbb{D}^{1, *}$ \\ Shan Liu (iD ${ }^{1, *}$ \\ Yutong Zhaol,* \\ Xiuhua Yang ${ }^{2}$
}

'Department of Clinical Medicine, China Medical University, Shenyang, Liaoning, People's Republic of China; ${ }^{2}$ Department of Obstetrics, The First Hospital of China Medical University, Shenyang, Liaoning, People's Republic of China

*These authors contributed equally to this work
Correspondence: Xiuhua Yang Department of Obstetrics, The First Hospital of China Medical University, Shenyang, Liaoning, People's Republic of China

Email yangxiuhuajing@hotmail.com

\begin{abstract}
Listeria monocytogenes (LM) is an intracellular, aerobic and facultative anaerobic, Gram-positive bacterium, which is primarily transmitted to humans orally via food. LM could occur in asymptomatic pregnant women; however, fetal infection is a serious condition, entailing premature birth, abortion, sepsis, central nervous system (CNS) involvement, or even death. If a pregnant woman exhibits symptoms, the performance is almost like influenza, such as fever, headache, diarrhea, myalgia, or other digestive-related symptoms. This review collected clinical and empirical results regarding the mechanism, clinical manifestations, obstetrical outcome, diagnosis, treatment, vertical transmission, neonatal infection, and prevention of listeriosi according to articles published in PubMed from January 1, 1980, to March 20, 2021. The early detection and diagnosis of pregnancy-associated listeriosis are significant since sensitive antibiotics are effective at enhancing the prognosis of newborns. Listeriosis can be diagnosed using positive cultures from maternal or neonatal blood, neonatal cerebrospinal fluid (CSF), amniotic fluid, intrauterine mucosa, or the placenta. Two weeks of high-dose intravenous amoxicillin (more than $6 \mathrm{~g}$ /day) is recommended for LM pregnant women without allergy. Terminating the pregnancy to save the mother's life should be considered if maternal and fetal conditions aggravate. Neonatal Listeria infection is primarily transmitted through the placenta, which is a critical illness associated with a high mortality rate. The necessary dietary guidance for pregnant women can reduce the incidence rate of pregnancy-related listeriosis.
\end{abstract}

Keywords: Listeria monocytogenes, listeriosis, pregnancy, neonate, infection, fever

\section{Introduction}

LM is an intracellular, aerobic and facultative anaerobic, Gram-positive bacterium, which was discovered accidentally in a prevalent infection in laboratory animals. ${ }^{1} \mathrm{LM}$ is primarily transmitted to humans orally via food, accounting for $99 \%$ of cases. ${ }^{2}$ Listeria has a higher likelihood to contaminate uncooked or ready-to-eat food, such as meat, vegetables, milk, and dairy products. ${ }^{3}$ LM can develop in the refrigerator over a long time and withstand large-scale temperatures $\left(-0.4-45^{\circ} \mathrm{C}\right){ }^{4}$ Reportedly, $\mathrm{LM}$ is more prevalent during the summer. ${ }^{5}$ Its incidence rate is considerably low, and it is preventable and treatable However, it is associated with high hospitalization and mortality rates $(20-50 \%){ }^{6}$ Serious consequences of listeria infection include gastrointestinal inflammation, sepsis, and central nervous system (CNS) involvement. ${ }^{7}$ From 1996 to 2005, LM accounted for $30 \%$ of lethal food-borne infections in the U. S. A, with a mortality of $16.9 \% .^{8}$ In 2015,2224 LM cases were reported in Europe, with a total mortality of $18.8 \%{ }^{9}$ Meanwhile, 253 LM patients were discovered across 19 provinces in China between 2011 and 2016, with a mortality rate of $25.7 \%$. $^{10}$ Fortunately, fatalities did not include pregnant females or children. ${ }^{10}$ 
According to the World Health Organization (WHO), the onset of Listeria during pregnancy accounted for nearly $43 \%$ of total cases, and $14 \%$ occurred in late pregnancies. ${ }^{11}$ Recently, published data for incidence index in pregnancyrelated listeriosis accounts for $11 \%$ of all listeriosis cases in Italy, ${ }^{12} 16 \%$ in Spain, ${ }^{13}$ and $17.7 \%$ in France. ${ }^{14}$ In France, the index declined from 60 to 5 cases in every 100,000 live births between 1984 and 2011, reducing by more than 12 times. ${ }^{15}$ In contrast, in Israel, this indicator increased from 5.5 to 25.2 cases in every 100,000 live births between 1998 and $2007 .{ }^{16}$ Moreover, $41.1-52 \%$ of listeriosis was associated with pregnancies in China, highlighting the nationwide pressure of this disease. ${ }^{17,18}$ Recently, the incidence rate of pregnancyrelated listeriosis shows declining statistics in many countries, such as France, Belgium, and the USA. ${ }^{15,19,20}$ Likely, the more stringent requirements in food production, special preventive measures, and better health care for pregnant females influenced the decline. ${ }^{15,19,20}$ Females with LM may not exhibit typical symptoms or have influenza-like symptoms, such as fever, irritating headache, or myalgia. ${ }^{21}$ However, Listeria could move through the placenta to affect the fetu. ${ }^{22}$ Besides, swallowing amniotic fluid can also be a route of fetal infection. ${ }^{23}$ Unlike mothers, fetal infection is a serious condition, entailing premature birth, abortion, sepsis, CNS involvement, or even death. ${ }^{24}$ Considering the seriousness of LM, timely anti-infection management must be initiated immediately upon diagnosing maternal-fetal LM; because prompt treatments can prevent adverse consequences. $^{25}$

Due to latent bad outcomes, it is crucial for obstetricians to familiarize themselves with the related information of listeria infection. This review aims to collect the clinical and empirical results regarding LM in pregnancies. Two independent researchers searched PubMed for articles published from January 1, 1980, to March 20, 2021, related to global patients with LM using the keywords "listeria", "listeriosis", "pregnancy", "maternal", and "neonate". A detailed aggregation of relevant studies on the mechanism, clinical manifestations, obstetrical outcome, diagnosis, treatment, vertical transmission, neonatal listeriosis, and prevention was performed.

\section{The Mechanism of Listeria in Pregnancy}

Once Listeria enters the human body through oral administration, it first arrives at the mucosa of the small intestine, and then enters other organs via the circulatory system and lymph nodes. Since Listeria is an intracellular bacterium, this scarce characteristic makes it easy to conceal in the host cells, making diagnosis difficult. Different proteins, such as certain internalins, are conducive for LM to attack host cells. Once the bacteria enters the cell, Listeria releases listeriolysins and phospholipases to dissolve the membrane of the vacuole to survive in cells. When LM reaches the cytoplasm, it can proliferate and elevate the development of actin filaments, making LM penetrate the cytoplasm and eventually arrive at the plasma membrane. Listeria usurps the host cell cytoskeleton, meaning that actin filaments it needs to move (not penetrate) through the cytoplasm are not produced by the bacteria. Listeria causes the polymerization of actin filaments from the infected cell in one of its poles, forming the well-known actin tail or comet that provides the ability of the bacteria to move inside the cell, finally reaching the plasma membrane and causing protrusions needed for intercellular transmission. Afterward, plasma membrane protrusions and intercellular diffusion infect neighboring cells. The circulation allows Listeria to avoid contacting the extracellular circumstance so that it can escape from the $\mathrm{T}$ cell immunity in humans by moving from one cell to another and continue to infect other organs and tissues. ${ }^{26}$ Moreover, antibodies, complement, and neutrophils also lose their protective effect on the host cells based on this specific intracellular circulation of LM. ${ }^{27}$ The virulence of LM depends on the infected subject's immunity, types of bacterial strains, and the infection scope. ${ }^{28} \mathrm{~A}$ dose of $10^{4}$ $10^{6}$ organisms/gram of food ingested can cause clinical $\mathrm{LM}^{22}$ However, for people at high risk of immunodeficiency, the dose needed for infection may be lower. ${ }^{22}$

Presently, 13 serological types of LM have been discovered. ${ }^{29}$ However, in most LM infections reported in Europe and many other nations, including China, just four types $(1 / 2 \mathrm{a}, 1 / 2 \mathrm{~b}, 1 / 2 \mathrm{c}$, and $4 \mathrm{~b})$ give rise to clinical LM infection. ${ }^{30}$ Serological-type $4 \mathrm{~b}$ has the strongest virulence, and could even cause a pandemic in humans, although type $1 / 2 \mathrm{a}$ is most commonly discovered in consumables. ${ }^{10}$ Compared to non-pregnant women, serotype $4 \mathrm{~b}$ has a higher likelihood to infect pregnant women. ${ }^{16,31}$ It is crucial to identify and eliminate LM from the infected person through innate and adaptive immunity. ${ }^{32}$ Compared to normal humans, pregnant females have 18 times higher likelihood of becoming infected by LM after ingesting contaminated consumables. ${ }^{1}$ It is partly due to reduced T cell immunity in pregnant females and insufficient decidual $\mathrm{T}$ cells due to 
epigenetic silence of chemical factors. The placental directionality (ActA and InlP virulent determinants) and the sheltered intracellular life circulation of LM trigger the spread of the bacteria to other parts of the body besides the digestive system. ${ }^{1,33-36}$ LM invades the placenta in two ways: direct invasiveness and cellular infection. ${ }^{37}$

Empirical studies have revealed that LM can invade both extravillous trophoblast cells (EVT) ${ }^{37}$ and syncytiotrophoblast (SYN).$^{38}$ Immunohistochemistry indicates that LM entered the SYN of the placenta in patients with listeriosis. ${ }^{38}$ Most infections occurred in the third trimester of pregnancy, further indicating that SYN could be where the bacteria enter the placenta. ${ }^{39}$ LM could immediately attach to the trophoblastic epithelium, enter the trophoblastic layer, and gain access to the center of villous tissues. According to the principle of kinetics, the bacteria initially infect the cells at the middle arterial catheter of the placenta, where the stain was positive for cytokeratin, indicating their fetal trophoblastic source. Afterward, the bacteria spread rapidly to other trophoblastic regions, including SYN cells at the villous center in the labyrinthine area of the placenta.

In empirical studies involving mice, pregnant mice were more likely to suffer from LM infection related to Foxp $^{+}{ }^{+}$regulatory $\mathrm{T}$ (Treg) cells. ${ }^{40}$ In pregnancy, LM enhances embryonic damage, gradually leading to fetal death, primarily by infecting the fetus, or secondarily via decreasing the fetal tolerance characteristic of Treg cells. ${ }^{41}$ The enhancement of inflammatory neutrophils and macrophages results in the accumulation of maternal $\mathrm{T}$ cells related to fetal antigens causing fetal death. The $\mathrm{CD} 8^{+}$ $\mathrm{T}$ cells elevated the CXCR3 level, accumulating $\mathrm{CD} 8^{+}$ $\mathrm{T}$ cells in the decidua and fetal death, ${ }^{42}$ which indicates that systemic listeriosis in pregnancy could be an immune imbalance related to placental infection. ${ }^{43}$ Compared to full-term neonates, preterm infants are highly vulnerable to LM due to reduced immune functionality. ${ }^{32}$ The association between internalin (InlA) and E-cadherin has a vital role in passing through the placental barrier for $\mathrm{LM}^{38}$ Also, fetal loss due to pregnancy-related LM was associated with the augment of CXCL9-releasing inflammatory neutrophils and macrophages in the placenta, which induced the transfer of fetal-specific $\mathrm{T}$ cells into the decidua. ${ }^{42}$ It was considered that dramatically reduced chemokines at maternal-fetal interface induced the etiology of pregnancy-related infection. Theoretically, incrementing these cytokines could be implemented as an efficient approach for alleviating immune-related pregnancy diseases. ${ }^{42}$ Reportedly, the anti-Listeria reactions at maternal-fetal interface relied on colonystimulating factor-1 (CSF-1)-mediated macrophages. ${ }^{44}$ Besides, LM infection diminished heme oxygenase (HO)1 and B-cell lymphoma-extra large (Bcl-XL) levels, which increased cell death in the placenta, ultimately leading to infective miscarriage. ${ }^{45}$ The schematic diagram of Listeria penetrating the placental barrier is shown in Figure 1.

\section{Maternal Clinical Manifestations of Listeria in Pregnancy}

Mothers infected with Listeria could be asymptomatic. If a pregnant woman exhibits symptoms, the performance is non-specific in most cases, almost like influenza, with symptoms such as fever, headache, diarrhea, myalgia, or other digestive-related symptoms. ${ }^{46,47}$ The literature reports outline that $65-81 \%$ of pregnant women have a fever, which is the most common clinical manifestation. ${ }^{5,46,48,49}$ The majority of infected pregnant women would exhibit a temperature above $38^{\circ} \mathrm{C}$, yet, mostly below $39^{\circ} \mathrm{C}$. Most importantly, some patients do not have feverish symptoms, and such cases challenge the diagnosis of clinicians. ${ }^{16,39,46}$ If LM access circulations system, the patient could develop sepsis, with poisoning symptoms, such as high fever, shiver, and breathing difficulties. The brain natriuretic peptide (BNP) could increase when cardiac function is involved. Sepsis could induce multiple organ failure or post-septic shock. However, the incidence of these two scenarios is low. Moreover, LM could cause chorioamnionitis. Meanwhile, the bacteria could hide in the placenta and cause periodic infections, increasing the difficulty to treat patients. ${ }^{50} \mathrm{LM}$ infection rarely leads to maternal death, yet, it is related to maternal primary diseases. ${ }^{16,51}$

In empirical studies involving mice, if pregnant mice were infected with LM on the 15th day of pregnancy, it would be challenging to remove the bacteria in vivo, which could lead to severe necrotizing hemorrhagic hepatitis in mice. ${ }^{52}$ The result indicates that pregnant females could also have liver involvement. During the physical inspection process, several patients reported percussion pain in bilateral renal areas, accompanied by urinary irritation symptoms, indicative of urinary tract infection or oncoming pyelonephritis. The chance of CNS involvement in normal pregnant females is extremely slight. In fact, most CNS lesions appear in mothers with suppressed immune systems. ${ }^{53}$ 


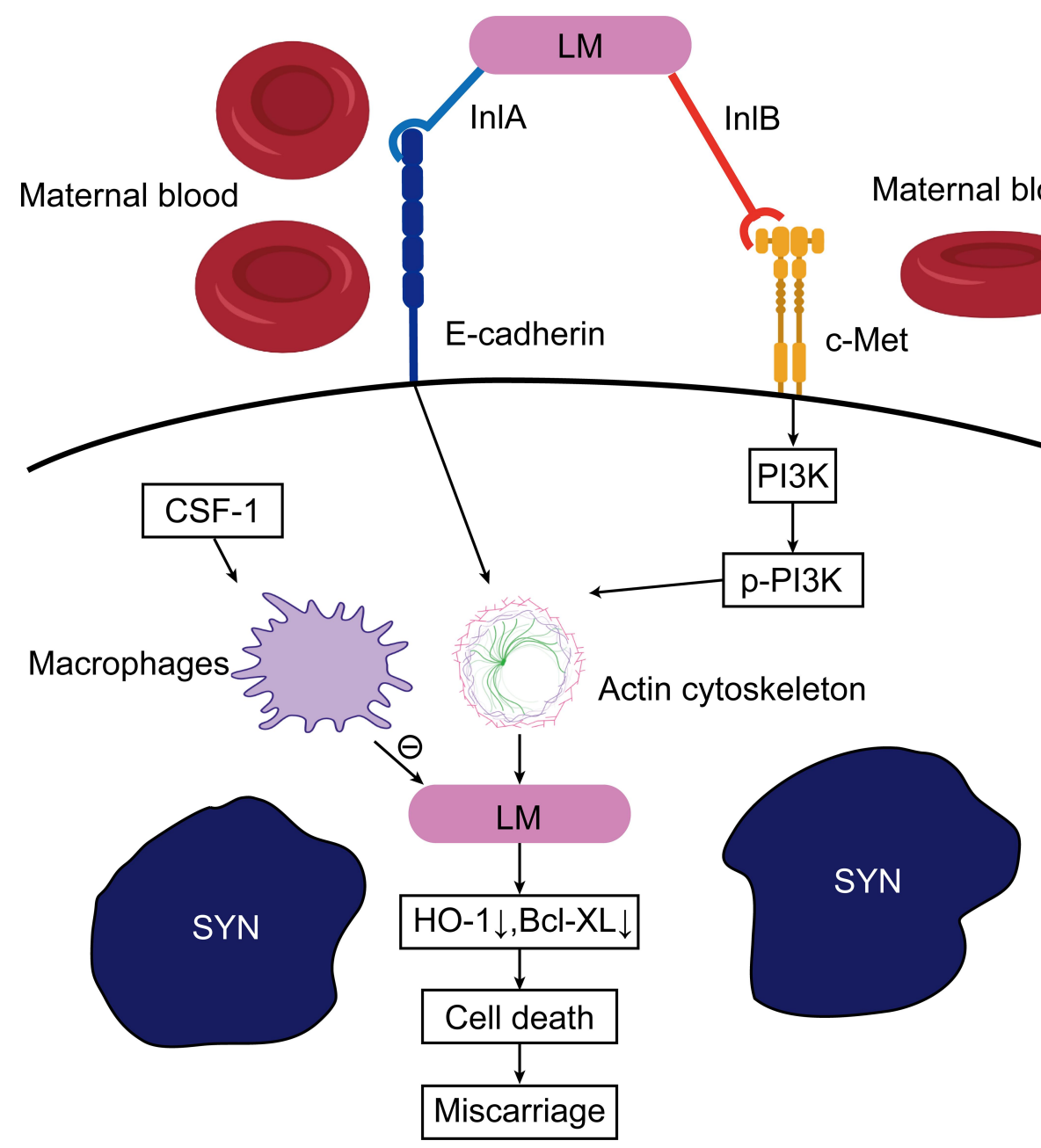

Figure I The schematic diagram of Listeria penetrating the placental barrier. The associations of InIA and InIB with their corresponding receptor E-cadherin and c-Met behave in a conjugated and codependent way to regulate the invasion of the placenta. InIB-c-Met activity induces PI3K. Thus, phosphorylated PI3K is formed in the syncytiotrophoblast (SYN), which is crucial for the realignments of the actin cytoskeleton. In these two ways, Listeria monocytogenes (LM) invades the SYN and reduces the expressions of heme oxygenase ( $\mathrm{HO})$-I and B-cell lymphoma-extra large (Bcl-XL), causing the cell death and miscarriage finally. Besides, colony-stimulating factor-I (CSF-I) stimulates macrophages against LM.

Therefore, pregnancy is not a high-risk index for maternal neurolisteriosis. However, CSF culture is a crucial consideration for pregnant women with immune deficiencies after LM infection. Especially, pregnant women could show signs of headache, suggesting the possibility of meningitis, cerebral thrombosis, or other neurological diseases. Infected pregnant women with meningitis could lose consciousness or exhibit other neurological symptoms.

The incubation time of LM could be considerably longer, which is unlike other foodborne illnesses. Based on the reported data, maternal-neonatal listeriosis has a rough incubation period of 19 to 27.5 days (scope: 7-67 days), which takes more time than neurolisteriosis (9 days; scope: 1-14 days), and bacteremia (2 days; scope: 1-12 days). ${ }^{54,55}$ It infers that it could take longer for the puerpera to exhibit bacteremia, placental involvement, and fetal infection before evolving. In 2002, a review was published on 11 pregnant women infected with LM. Ten of them had blood routine tests, and eight of them had considerably elevated white blood cells (WBC), ${ }^{46}$ which conformed with previous studies. ${ }^{56,57}$ However, for normal pregnant women, the WBC number could increase from $6 \times 10^{9} / \mathrm{L}$ in early pregnancy to $16 \times 10^{9} / \mathrm{L}$ during the third trimester. ${ }^{11}$ Therefore, the number of WBC needs to be compared with this index's fundamental level. If there is a progressive rise in the numbers of $\mathrm{WBC}$, obstetricians should consider the possibility of an LM infection.

\section{Obstetrical Outcome of Listeria in Pregnancy}

Mothers infected with Listeria may be aware of reduced fetal movement and noticeable uterine contractions, or 
symptoms of threatened preterm birth, such as abdominal pain, vaginal bleeding, or premature rupture of membrane. The non-stress test shows that the baseline of fetal heart beating was over $160 \mathrm{bpm}$, without any significant variation or only slight variation. Maternal infection with LM could lead to chorioamnionitis or meconium-like amniotic fluid during delivery. Apart from above-mentioned presentations, the literature also reports that abnormalities of fetal digestive tract are discoverable through an ultrasound, including fetal ascites, gallbladder enlargement, intestinal echo enhancement, and small intestine widening, implying the likelihood for a fetal intrauterine infection. ${ }^{58}$ The newborn was born with moderate jaundice and dyspnea in this case. $^{58}$

The gestational age at the time of infection has a fundamental influence on the prognosis of newborns. ${ }^{15}$ If the fetal infection is diagnosed in early pregnancy, $65 \%$ of the pregnant women have abortions. ${ }^{51}$ If the infection occurs during the second or third trimester of pregnancy, $26 \%$ of cases could end up in stillbirths, uterine fetal loss, or abortion. ${ }^{51}$ LM tends to occur in late pregnancy. ${ }^{59}$ However, there were also confirmed cases in early pregnancy. ${ }^{60,61}$ The reason for the lower incidence of LM in early pregnancy may be that the culture of the embryo or maternal blood is rarely conducted after pregnancy loss. ${ }^{22}$ Therefore, it is crucial to follow up the medical history and laboratory examination of patients with early spontaneous abortions.

Regarding maternal listeriosis, just $5 \%$ of pregnancies have favorable pregnancy outcomes. ${ }^{39}$ The MONALISA group report confirmed that $82 \%$ of pregnant women faced severe consequences (88/107), such as fetal loss $(25 \%, 27 /$ $107)$, premature birth before 32 gestational weeks (19\% in the maternal group and $42 \%$ in all premature neonates); moreover, there were also some newborns with early-onset or late-onset listeriosis. ${ }^{39}$ Furthermore, although accepting proposals for preemptive ampicillin treatment for maternal fever, the incidence of fetal loss associated with LM has not reduced over the recent years. ${ }^{16,39,46,62}$ Reportedly, in a research that involved 166 fetal listeriosis cases, the neonatal survival rate was $0,29.2 \%$, and $95.3 \%$ during the early, middle, and late pregnancies, respectively. ${ }^{16}$ From 1967 to 1985 , the net perinatal mortality rate of listeriosis in Britain was around $50 \%$ among 722 cases. $^{62}$ Since 2004, Listeria infection monitoring across 10 regions of the USA shows that $17 \%$ of 760 Listeria infection cases were related to pregnancies, with a $29 \%$ incidence rate for fetal loss and neonatal fatalities. ${ }^{63}$ Improved perinatal outcomes could be related to the development of treatment capacity in neonatology and a better understanding of pregnancy-related listeriosis.

The criticalness of the influence on the fetus changed with the occurrence of maternal clinical presentations. According to a study performed in England and Wales from 1990 to 2010, pregnant women exhibiting symptoms are more likely to give birth to a stillbirth or have spontaneous miscarriage. ${ }^{64}$ It could be because most pregnant women exhibiting symptoms are in the early or second trimester of pregnancy, or an excessive quantity of Listeria exists in the body. Empirical guinea pig studies have confirmed that it takes nine days from the onset to the death of the fetus. ${ }^{65}$ The intermediate delay interval indicates that the bacteria need to colonize the placenta before infecting the fetus. In humans, although undiagnosed, LM might invade the placenta and cause intrauterine infection in asymptomatic parturient cases.

In China, there were 12 cases of pregnancy-related LM infection in a hospital for five years since 2013, including 10 singletons and 2 twins. $^{23}$ The incidence of pregnancyassociated Listeria infection was 13.7/100,000 deliveries. $^{23}$ All mothers were cured. ${ }^{23}$ Unfortunately, two spontaneous abortions and four fetal losses occurred. $^{23}$ Eight neonates were born, six were normal, and two postnatal deaths occurred within 48 hours. $^{23}$ Postnatal assessment of the surviving newborns did not reveal any neurological complications. ${ }^{23}$ The total fetalneonatal mortality was $57.1 \%$; importantly, this indicator was $100 \%$ for infections discovered in the second trimester of pregnancy, and just $14.3 \%$ for those happening in the third trimester. ${ }^{23}$

\section{Diagnosis of Listeriosis in Pregnancy}

The early detection and diagnosis of pregnancy-associated listeriosis are significant since sensitive antibiotics effectively enhance the prognosis of newborns. ${ }^{22,25}$ When pregnant females exhibit unexplained fever, physicians are more likely to think of listeriosis. ${ }^{16,39}$ In particular, when females have a record of consuming potentially contaminated foods within the last month ${ }^{46}$ or in a scenario where there is comprehensive information on various salient problems, including smoking or alcohol consumption. ${ }^{66}$ Listeriosis can be diagnosed using positive cultures from sterile samples. Clinically, isolation is generally from maternal or neonatal blood, neonatal CSF, amniotic fluid, intrauterine mucosa, or the placenta. ${ }^{22}$ The diagnosis of listeria infection primarily depends on blood culture. If the 
blood culture is negative and the suggested antibiotics are taken, the decision of whether to continue using antibiotics should be based on the opinions of infectious experts, high-risk obstetricians, and neonatal physicians. Besides, the development of LM takes 36 hours. Meanwhile, the proportion of positive blood cultures among symptomatic females is only $36-55 \%$. $^{25,39,46}$ Additional test results, including increased WBC, vaginal smear, or Gram stain, could also be useful. ${ }^{46,67-69}$

Swift discrimination and discovery of LM are also probable, with the help of serological detection for antilisteriolysin, which was secreted by the host against the bacterium listeriolysin $\mathrm{O}$ (LLO). ${ }^{22,70}$ The host secretes immunoglobulins that pertain to serotype $G$, while the IgM antibody tends to exist temporarily. Anti-LLO IgGs are positive for a few days after the infection and can last for several months even after the parturient completes treatment with sensitive antibiotics, which differs from the blood culture. ${ }^{71}$ The positive rate of $\operatorname{IgG}$ antibody in sera of females with a history of abortion was $35.6 \%$ (89/ $250)$, while that was $17.5 \%(35 / 200)$ in women with a normal childbirth $(P=0.001)$, suggesting that $\mathrm{LM}$ was related to spontaneous miscarriage. ${ }^{72}$ The test of anti-LLO antibodies could be a vital means for serological monitoring if pregnant women with a high-risk factor of listeriosis show obvious symptoms. On the contrary, it is not suggested for all pregnant women, in particular for females with atypical symptoms, because the positive rate of serological antibody is very high in the general population. ${ }^{73}$ The serum antibody could still be positive for the patients who have a history of LM infection or have been systematically treated, causing unnecessary confusion and stressing the pregnant women and their physicians. Besides, the obstetrician would find it challenging to deal with such a positive result because it cannot be diagnosed or ruled out immediately.

Bacterial culture of placental tissues is the most sensitive method to diagnose maternal-neonatal listeriosis. ${ }^{39} \mathrm{It}$ should be performed in combination with maternal blood culture. ${ }^{39}$ The positive rates of the two tests are $80 \%$ and $55 \%$, respectively. ${ }^{39}$ The culture of neonatal gastric aspirates is also beneficial to determine whether a neonatal infection exists. ${ }^{39}$ Placental cultures should be acquired during delivery. Retrospective placental detection helps detect additional Listeria infections; however, this pathological biopsy was performed in only half of neonatal infections. ${ }^{74}$ Following Listeria infection, specific changes could occur in placental tissues, such as extensive abscesses, or necrosis in small blood vessels. ${ }^{75}$ Besides the placental examination, amniotic fluid can be extracted using amniocentesis. If gram-positive rods are detected in amniotic fluid, it could be an indicator of Listeria infection, which is another rapid detection technique. ${ }^{76}$ The result of amniotic fluid is also useful as an auxiliary diagnostic method to guide the treatment. ${ }^{77}$ In cases that lack conclusive evidence of maternal-fetal listeriosis, the listeriosis culture from non-invasive cervical/vaginal smears could contribute to the diagnosis of fetal listeriosis. ${ }^{5,78}$ Recent research revealed that the positive rate of culture for cervical or vaginal smears was $26 \%$ in maternal-neonatal listeriosis cases. ${ }^{39}$ In Iran, 5.5\% (22/ 400) of vaginal smear samples were confirmed to be culture positive for LM in normal pregnant females. ${ }^{79}$ Accordingly, vaginal smear culture can be considered for pregnant women with high-risk factors during the regular prenatal examination since this examination is nontraumatic, easy to operate, and can improve the detection rate of LM to avoid adverse consequences.

Presently, stool culture is not recommended to detect Listeria due to numerous Listeria-containing substances in the environment. Therefore, the possibility of ingestion of LM and the presence of listeriosis in feces is also high. Thus, intermittent bacteria carrying or shedding in feces (about $5 \%$ in the general population, but a huge difference occurs) rarely indicates of an infection. ${ }^{22}$ Moreover, a fecal culture of LM is less sensitive, and most laboratories are ill-equipped to do tests.

\section{The Treatment of Listeria in Pregnancy}

Two weeks of high-dose intravenous amoxicillin (more than $6 \mathrm{~g} /$ day) is recommended for LM patients without allergy. Amoxicillin's safety for the fetus has been verified conclusively. ${ }^{80,81}$ It is also suitable if the remedy continues to the puerperium or starts after delivery. Mainly because it is safe for newborns during breastfeeding, the relative infant dose (RID) (the ratio of the drug concentration in neonatal blood to that in maternal blood) of amoxicillin is only $0.2-0.5 \%{ }^{82}$ Typically, adding gentamicin to the medication scheme is also possible, because studies have shown that gentamicin has a synergistic effect with amoxicillin. ${ }^{83}$ However, some specialists have questioned this usage, primarily based on the toxicity of gentamicin to the fetus. ${ }^{5}$ Gentamicin combined with ampicillin/amoxicillin could improve the survival rate of invasive LM; 
however, it does not affect intracellular Listeria in macrophages. ${ }^{39,84}$ Typically, cephalosporin is commonly administered to pregnant women to prevent or cure infectious diseases, with a significant effect on group B Streptococcus and Escherichia coli. However, it does not affect LM. ${ }^{16,25,74}$

Clinical treatment is difficult if the pregnant woman is allergic to penicillin or amoxicillin. In such cases, trimethoprim with sulfamethoxazole is a secondary line of medication. ${ }^{5}$ However, trimethoprim could damage the fetal heart and nervous system during early pregnancy. ${ }^{85}$ In that case, erythromycin is suitable for patients allergic to penicillin, as it does not harm the fetus. ${ }^{86,87}$ The only limitation of erythromycin is that its concentration reduces after passing through the placenta. Thus, the dosage needs to be increased. Erythromycin is also available for breastfeeding. ${ }^{88}$ Generally, 2-3 weeks of treatment is sufficient for different serotypes of Listeria. However, treatment is needed for 4 weeks if the maternal nervous system is involved. ${ }^{5,83}$

Limited literature exists regarding drug resistance in the treatment of LM in pregnant females. One study reported extremely high levels of drug resistance for clindamycin (66.7\%), penicillin G $(66.7 \%)$, amoxicillin $(50 \%)$, and vancomycin $(50 \%){ }^{88}$ Thus, high drug resistivity for medication to manage listeriosis poses a significant risk to the clinical outcome of Listeriosis-associated pregnancies. In contrast, LM separated from patients with spontaneous miscarriage in Iran was $100 \%$ sensitive to trimethoprim and erythromycin and showed higher susceptibility to chloramphenicol $(88 \%)$ and ciprofloxacin $(66.67 \%) .{ }^{89}$ Moreover, in Poland, it was found that listeria detected in all instant consumable foods were susceptible to ciprofloxacin, chloramphenicol, and trimethoprim/

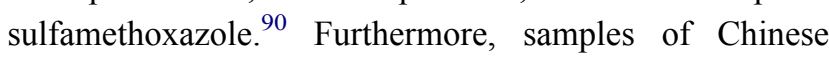
food exhibited high sensitivity to ciprofloxacin (90.5\%) and trimethoprim/sulfamethoxazole $(57.1 \%){ }^{91}$

There is controversy around the possibility of vaccination against Listeria infection during pregnancy. Prenatal vaccination with the attenuated LM strains before or during pregnancy cannot prevent fetal loss or maternal-fetal listeriosis. $^{92}$ However, some reports indicate that nano vaccines show potential. $^{93}$

Considering that timely and sufficient antibiotic treatment can improve the prognosis of mothers and infants significantly, certain physicians have suggested that the prophylactic use of antibiotics should be considered among pregnant women with fever and/or gastrointestinal symptoms. ${ }^{94}$ It has been suggested that orally administering amoxicillin or trimethoprim-sulfamethoxazole for one week during the second or the third trimester is useful for high-risk pregnant women suspected of Listeria infection. ${ }^{95}$ However, in 2011 , centers for disease control and prevention (CDC) published a recommended schedule for clinically treating individuals facing an elevated risk of invasive listeriosis after consuming foods containing LM. $^{96}$ The specialists highlighted that there is no medical indication for detection and preventive treatment of suspected cases of LM infection, because their probability of developing into invasive Listeria is rather low. ${ }^{96}$ Accordingly, the American College of Obstetricians and Gynecologists (ACOG) stated that there is no indication to perform blood culture, stool culture, or treatments for pregnant women who do not show any obvious symptoms, despite their food was recalled or they were involved in the epidemic of Listeria infection. ${ }^{21}$ These views were also agreed upon in subsequent studies following Listeria outbreaks in many locations. ${ }^{96}$

Terminating the pregnancy to save the mother's life should be considered if maternal and fetal conditions aggravate. Moreover, if the pregnant female has systemic infections, such as cardiovascular system involvement, or liver and kidney dysfunctions, the pregnancy needs to be terminated to save the female. Another case that would necessitate terminating the pregnancy is if the placental function decreased or non-stress test showed repeated variant deceleration or late deceleration. ${ }^{46,62,68}$ Considering the above scenarios, obstetricians should improve awareness regarding Listeria in pregnancy, and early and timely antibiotic treatment is essential to control the disease. Simultaneously, patients should be tested accordingly.

\section{Vertical Transmission of Listeriosis}

Neonatal Listeria infection is primarily transmitted through the placenta and rarely infected through close contact after childbirth or nosocomial infection. ${ }^{16,64,75}$ In one case, a pregnant female at her 31 gestational weeks became infected with listeriosis, and micro-abscess changes appeared in the endometrium, unfortunately, the neonate had meningitis and bacteraemia. ${ }^{97}$ Molecular detection via random amplified polymorphic DNA (RAPD) discovered that there are three identical strains in maternal blood and neonatal blood, providing strong evidence of vertical transmission. ${ }^{97}$ In another case of neonatal septicemia after infection with Listeria, the positive blood culture result was a further indication of the 
existence of vertical infection. ${ }^{98}$ If the pregnant woman was infected with LM with asymptomatic symptoms, the newborn may get infected by this bacteria through the birth canal, showing late-onset listeriosis, leading to neurological symptoms. ${ }^{99}$

\section{Neonatal Listeriosis}

Neonatal listeriosis is a critical illness associated with a high mortality rate. ${ }^{71}$ Based on clinical manifestations, neonatal listeriosis can be divided into two types: early onset disease (EOD) and late onset disease (LOD). ${ }^{62}$ Generally, EOD happens within 6 days after birth, and pregnant women often have slight clinical manifestations, while neonates may exhibit clinical characteristics including dyspnea, pneumonia, septicemia, or cephalomeningitis. ${ }^{100}$ The mortality of EOD is around $20 \%$, and follow-up research showed that $40 \%$ of survivors had neurological complications. ${ }^{22}$ LOD occurs 7-28 days after birth, which is commonly diagnosed in full-term newborns with asymptomatic mothers. ${ }^{1}$ LOD neonates might develop sepsis or meningitis with a mortality rate of $10 \% .{ }^{1}$ LOD patients could also have critical complications, such as constrained physical development or nervous system disorders, even after they were cured. ${ }^{100}$ Reportedly, there is a $68 \%$ probability of neonatal sepsis due to Listeria infection. ${ }^{22}$ Consequently, newborns born to infected mothers require follow-up tests until 2-3 months after delivery.

Manifestations of the nervous system, such as neonatal Listeria meningitis, are relatively common. Researchers have discovered that the primary cause of neonatal death is younger gestational weeks at birth, rather than the appearance of LM in neonatal CSF, indicating that other issues, such as preterm delivery affect mortality. ${ }^{15,64}$ Besides, the probability of death caused by Listeria meningitis reduces as the newborn becomes older. Compared to the newborn within 15 days after birth, the mortality of newborns aged 15-30 days was remarkably lower. ${ }^{51}$ In England, $81.4 \%$ of newborns with neurological dysfunction survived. ${ }^{64}$ In France, $94 \%$ of newborns were diagnosed as EOD, and $5 \%$ as LOD. The mortality of the EOD cases was $8 \%$, while there were no fatalities in LOD. Unexpectedly, LM was positive in all neonatal CSF among LOD cases. ${ }^{15}$ These reports showed that age is a principal indicator in the survival of newborns with Listeria infection.

\section{Prevention of Listeriosis in Pregnancy}

Generally, most pregnant females infected with LM had consumed some form of food that posed a risk to the pregnancy. It stresses the importance of providing health education for pregnant women in areas with a high incidence of LM. Pregnant women should refrain from having ready-to-eat food and dairy products without hightemperature disinfection. Concurrently, cross-infection should be refrained by ensuring that tableware is clean and tidy and the tabletop used for food preparation is free from LM contamination. From a seasonal perspective, there is a certain time lag between the seasonal peak of pregnancy-related listeriosis and other listeriosis. Furthermore, physicians should inform all pregnant females about symptoms of listeriosis so that they can seek the help of doctors promptly after clinical manifestations appear. Fortunately, the French government has taken measures to control Listeria contamination in the process of food processing, consequently, from 1984 to 2006, the Listeria infection rate decreased significantly. ${ }^{15}$ The necessary dietary guidance for pregnant women can reduce the incidence rate of pregnancy-related listeriosis. Importantly, the incidence of fetal death can be reduced by improving maternal awareness of Listeria infection. ${ }^{15}$

\section{Conclusions}

As Listeria is an intracellular bacterium, it is difficult to clinically diagnose and deal with it. It requires obstetricians to think about the possibility of pregnancy-related LM in pregnant women with influenza-like symptoms and highrisk diet history. Once pregnant females develop typical manifestations or other symptoms that raise suspiciousness about listeriosis, they should be tested for the bacteria and preventive medication should be administered to avoid bad maternal and fetal outcomes. Governments may need to strengthen the surveillance for listeriosis to reduce the incidence rate. Additional research direction is warranted to investigate the costs and benefits of routinely monitoring pregnant females for LM to decrease illness load and ameliorate prognosis through prophylactic management.

\section{Funding}

This work was supported by the Natural Science Foundation of Liaoning Province [grant number 2020MS-167]. 


\section{Disclosure}

The authors report no conflicts of interest in this work.

\section{References}

1. Mateus T, Silva J, Maia RL, Teixeira P. Listeriosis during pregnancy: a public health concern. ISRN Obstet Gynecol. 2013;2013:851712. doi:10.1155/2013/851712

2. Scallan E, Hoekstra RM, Angulo FJ, et al. Foodborne illness acquired in the United States-major pathogens. Emerg Infect Dis. 2011;17(1):7-15. doi:10.3201/eid1701.P11101

3. Farber JM, Peterkin PI. Listeria monocytogenes, a food-borne pathogen. Microbiol Rev. 1991;55(3):476-511. doi:10.1128/ MR.55.3.476-511.1991

4. Pagliano P, Arslan F, Ascione T. Epidemiology and treatment of the commonest form of listeriosis: meningitis and bacteraemia. Infez Med. 2017;25(3):210-216.

5. Janakiraman V. Listeriosis in pregnancy: diagnosis, treatment, and prevention. Rev Obstet Gynecol. 2008;1(4):179-185.

6. Swaminathan B, Gerner-Smidt P. The epidemiology of human listeriosis. Microbes Infect. 2007;9(10):1236-1243. doi:10.1016/ j.micinf.2007.05.011

7. Doganay M. Listeriosis: clinical presentation. FEMS Immunol Med Microbiol. 2003;35(3):173-175. doi:10.1016/S09288244(02)00467-4

8. Barton Behravesh C, Jones TF, Vugia DJ, et al. Deaths associated with bacterial pathogens transmitted commonly through food: foodborne diseases active surveillance network (FoodNet), 1996-2005. J Infect Dis. 2011;204(2):263-267. doi:10.1093/ infdis/jir263

9. Van Walle I, Björkman JT, Cormican M, et al. Retrospective validation of whole genome sequencing-enhanced surveillance of listeriosis in Europe, 2010 to 2015. Euro Surveill. 2018;23 (33). doi:10.2807/1560-7917.ES.2018.23.33.1700798

10. Li W, Bai L, Fu P, Han H, Liu J, Guo Y. The epidemiology of listeria monocytogenes in China. Foodborne Pathog Dis. 2018;15 (8):459-466. doi:10.1089/fpd.2017.2409

11. Wadhwa Desai R, Smith MA. Pregnancy-related listeriosis. Birth Defects Res. 2017;109(5):324-335. doi:10.1002/bdr2.1012

12. Mammina C, Parisi A, Guaita A, et al. Enhanced surveillance of invasive listeriosis in the Lombardy region, Italy, in the years 2006-2010 reveals major clones and an increase in serotype $1 / 2 \mathrm{a}$ BMC Infect Dis. 2013;13(1):152. doi:10.1186/1471-2334-13-152

13. Nolla-Salas J, Bosch J, Gasser I, et al. Perinatal listeriosis: a Population-Based Multicenter Study in Barcelona, Spain (1990-1996). Am J Perinatol. 1998;15(8):461-467. doi:10.1055/ s-2007-994067

14. Goulet V, Hebert M, Hedberg C, et al. Incidence of listeriosis and related mortality among groups at risk of acquiring listeriosis. Clin Infect Dis. 2012;54(5):652-660. doi:10.1093/cid/cir902

15. Girard D, Leclercq A, Laurent E, Lecuit M, de Valk H, Goulet V. Pregnancy-related listeriosis in France, 1984 to 2011, with a focus on 606 cases from 1999 to 2011. Euro Surveill. 2014;19(38). doi:10.2807/1560-7917.ES2014.19.38.20909

16. Elinav H, Hershko-Klement A, Valinsky L, et al. Pregnancyassociated listeriosis: clinical characteristics and geospatial analysis of a 10-year period in Israel. Clin Infect Dis. 2014;59 (7):953-961. doi:10.1093/cid/ciu504

17. Fan Z, Xie J, Li Y, Wang H. Listeriosis in mainland China: a systematic review. Int $J$ Infect Dis. 2019;81:17-24. doi:10.1016/j.ijid.2019.01.007

18. Feng Y, Wu S, Varma JK, Klena JD, Angulo FJ, Ran L. Systematic review of human listeriosis in China, 1964-2010. Trop Med Int Health. 2013;18(10):1248-1256. doi:10.1111/ tmi. 12173
19. Tappero JW, Schuchat A, Deaver KA, Mascola L, Wenger JD. Reduction in the incidence of human listeriosis in the United States. Effectiveness of prevention efforts? The Listeriosis Study Group. JAMA. 1995;273(14):1118-1122. doi:10.1001/ jama.1995.03520380054035

20. Bertrand S, Ceyssens PJ, Yde M, et al. Diversity of Listeria monocytogenes strains of clinical and food chain origins in Belgium between 1985 and 2014. PLoS One. 2016;11(10): e0164283. doi:10.1371/journal.pone.0164283

21. Committee on Obstetric Practice. Committee opinion no. 614: management of pregnant women with presumptive exposure to Listeria monocytogenes. Obstet Gynecol. 2014;124 (6):1241-1244. doi:10.1097/01.AOG.0000457501.73326.6c

22. Lamont RF, Sobel J, Mazaki-Tovi S, et al. Listeriosis in human pregnancy: a systematic review. J Perinat Med. 2011;39 (3):227-236. doi:10.1515/jpm.2011.035

23. Li $\mathrm{C}$, Zeng $\mathrm{H}$, Ding $\mathrm{X}$, et al. Perinatal listeriosis patients treated at a maternity hospital in Beijing, China, from 2013-2018. BMC Infect Dis. 2020;20(1):601. doi:10.1186/ s12879-020-05327-6

24. de Noordhout CM, Devleesschauwer B, Angulo FJ, et al. The global burden of listeriosis: a systematic review and meta-analysis. Lancet Infect Dis. 2014;14(11):1073-1082. doi:10.1016/S1473-3099(14)70870-9

25. Charlier C, Goffinet F, Azria E, Leclercq A, Lecuit M. Inadequate management of pregnancy-associated listeriosis: lessons from four case reports. Clin Microbiol Infect. 2014;20(3):246-249. doi:10.1111/1469-0691.12281

26. Pizarro-Cerdá J, Cossart P. Subversion of cellular functions by Listeria monocytogenes. $J$ Pathol. 2006;208(2):215-223. doi: $10.1002 /$ path. 1888

27. Hof H, Nichterlein T, Kretschmar M. Management of listeriosis. Clin Microbiol Rev. 1997;10(2):345-357. doi:10.1128/CMR.10.2.345

28. Schlech WF 3rd, Lavigne PM, Bortolussi RA, et al. Epidemic listeriosis-evidence for transmission by food. $N \mathrm{Engl} \mathrm{J} \mathrm{Med}$. 1983;308(4):203-206. doi:10.1056/NEJM198301273080407

29. Braga V, Vázquez S, Vico V, et al. Prevalence and serotype distribution of Listeria monocytogenes isolated from foods in Montevideo-Uruguay. Braz J Microbiol. 2017;48(4):689-694. doi:10.1016/j.bjm.2017.01.010

30. Li A, Xu H, Li X, et al. Genomic and phenotypic diversity of listeria monocytogenes causing pregnancy-associated listeriosis from Zhejiang Province, China, 2016-2018. Infect Drug Resist. 2020;13:1179-1184. doi:10.2147/IDR.S245305

31. Soni DK, Singh DV, Dubey SK. Pregnancy - associated human listeriosis: virulence and genotypic analysis of Listeria monocytogenes from clinical samples. J Microbiol. 2015;53(9):653-660. doi:10.1007/s12275-015-5243-9

32. Zenewicz LA, Shen $H$. Innate and adaptive immune responses to Listeria monocytogenes: a short overview. Microbes Infect. 2007;9(10):1208-1215. doi:10.1016/j. micinf.2007.05.008

33. Kourtis AP, Read JS, Jamieson DJ. Pregnancy and infection. $N$ Engl J Med. 2014;370(23):2211-2218. doi:10.1056/ NEJMra1213566

34. Nancy P, Tagliani E, Tay CS, Asp P, Levy DE, Erlebacher A. Chemokine gene silencing in decidual stromal cells limits $\mathrm{T}$ cell access to the maternal-fetal interface. Science. 2012;336 (6086):1317-1321. doi:10.1126/science. 1220030

35. Faralla C, Rizzuto GA, Lowe DE, et al. InlP, a new virulence factor with strong placental tropism. Infect Immun. 2016;84 (12):3584-3596. doi:10.1128/IAI.00625-16

36. Vigliani MB, Bakardjiev AI. Intracellular organisms as placental invaders. Fetal Matern Med Rev. 2014;25(3-4):332-338. doi:10.1017/S0965539515000066 
37. Robbins JR, Skrzypczynska KM, Zeldovich VB, Kapidzic M, Bakardjiev AI, Schneider DS. Placental syncytiotrophoblast constitutes a major barrier to vertical transmission of Listeria monocytogenes. PLoS Pathog. 2010;6(1):e1000732. doi:10.1371/journal.ppat.1000732

38. Lecuit M, Nelson DM, Smith SD, et al. Targeting and crossing of the human maternofetal barrier by Listeria monocytogenes: role of internalin interaction with trophoblast E-cadherin. Proc Natl Acad Sci U S A. 2004;101(16):6152-6157. doi:10.1073/ pnas.0401434101

39. Charlier C, Perrodeau É, Leclercq A, et al. Clinical features and prognostic factors of listeriosis: the MONALISA National Prospective Cohort Study. Lancet Infect Dis. 2017;17 (5):510-519.

40. Rowe JH, Ertelt JM, Aguilera MN, Farrar MA, Way SS. Foxp3 $(+)$ regulatory $\mathrm{T}$ cell expansion required for sustaining pregnancy compromises host defense against prenatal bacterial pathogens. Cell Host Microbe. 2011;10(1):54-64. doi:10.1016/j. chom.2011.06.005

41. Rowe JH, Ertelt JM, Xin L, Way SS. Listeria monocytogenes cytoplasmic entry induces fetal wastage by disrupting maternal Foxp3+ regulatory T cell-sustained fetal tolerance. PLoS Pathog. 2012;8(8):e1002873. doi:10.1371/journal.ppat.1002873

42. Chaturvedi V, Ertelt JM, Jiang TT, et al. CXCR3 blockade protects against Listeria monocytogenes infection-induced fetal wastage. J Clin Invest. 2015;125(4):1713-1725. doi:10.1172/ JCI78578

43. Krishnan L, Pejcic-Karapetrovic B, Gurnani K, Zafer A, Sad S. Pregnancy does not deter the development of a potent maternal protective CD8+ T-cell acquired immune response against Listeria monocytogenes despite preferential placental colonization. Am J Reprod Immunol. 2010;63(1):54-65. doi:10.1111/j.1600-0897.2009.00766.x

44. Qiu X, Zhu L, Pollard JW. Colony-stimulating factor-1-dependent macrophage functions regulate the maternal decidua immune responses against Listeria monocytogenes infections during early gestation in mice. Infect Immun. 2009;77(1):85-97. doi:10.1128/IAI.01022-08

45. Tachibana M, Hashino M, Nishida T, Shimizu T, Watarai M. Protective role of heme oxygenase-1 in Listeria monocytogenes-induced abortion. PLoS One. 2011;6(9):e25046. doi:10.1371/journal.pone.0025046

46. Mylonakis E, Paliou M, Hohmann EL, Calderwood SB, Wing EJ. Listeriosis during pregnancy: a case series and review of 222 cases. Medicine (Baltimore). 2002;81(4):260-269. doi:10.1097/ 00005792-200207000-00002

47. Jackson KA, Iwamoto M, Swerdlow D. Pregnancy-associated listeriosis. Epidemiol Infect. 2010;138(10):1503-1509. doi:10.1017/S0950268810000294

48. Sisó C, Goncé A, Bosch J, Salvia MD, Hernández S, Figueras F. Listeriosis in pregnancy: a secular trend in a tertiary referral hospital in Barcelona. Eur J Clin Microbiol Infect Dis. 2012;31 (9):2125-2132. doi:10.1007/s10096-012-1545-6

49. Larraín de la CD, Abarzúa CF, Jourdan HF, Merino OP, Belmar JC, García CP. [Listeria monocytogenes infection in pregnancy: experience of Pontificia Universidad Católica de Chile University Hospital]. Rev Chilena Infectol. 2008;25 (5):336-341. Infecciones por Listeria monocytogenes en mujeres embarazadas: experiencia del Hospital Clínico de la Pontificia Universidad Católica de Chile. Spanish. doi:10.4067/S071610182008000500003

50. Bakardjiev AI, Theriot JA, Portnoy DA. Listeria monocytogenes traffics from maternal organs to the placenta and back. PLoS Pathog. 2006;2(6):e66. doi:10.1371/journal.ppat.0020066
51. Silk BJ, Mahon BE, Griffin PM, et al. Vital signs: listeria illnesses, deaths, and outbreaks-United States, 2009-2011. MMWR Morb Mortal Wkly Rep. 2013;62(22):448-452.

52. Abram M, Schlüter D, Vuckovic D, Waber B, Doric $M$, Deckert M. Effects of pregnancy-associated Listeria monocytogenes infection: necrotizing hepatitis due to impaired maternal immune response and significantly increased abortion rate. Virchows Arch. 2002;441(4):368-379. doi:10.1007/s00428-0020649-2

53. Lu B, Yang J, Gao C, et al. Listeriosis cases and genetic diversity of their L. monocytogenes isolates in China, 2008-2019. Front Cell Infect Microbiol. 2021;11:608352. doi:10.3389/ fcimb.2021.608352

54. Angelo KM, Jackson KA, Wong KK, Hoekstra RM, Jackson BR. Assessment of the incubation period for invasive listeriosis. Clin Infect Dis. 2016;63(11):1487-1489. doi:10.1093/cid/ciw569

55. Goulet V, King LA, Vaillant V, de Valk $H$. What is the incubation period for listeriosis? BMC Infect Dis. 2013;13(1):11. doi:10.1186/1471-2334-13-11

56. Wu L, Zhang XH, Chen H, Yin XL. [Neonatal septicemia caused by Listeria monocytogenes: report of 6 cases]. Zhonghua Er Ke Za Zhi. 2008;46(1):22-25. Chinese.

57. Wang $\mathrm{P}$, Chen $\mathrm{Y}$, Wang $\mathrm{H}$, Yang $\mathrm{S}, \mathrm{Xu} \mathrm{Y}, \mathrm{Li} \mathrm{T}$. [A clinical analysis of 16 patients with maternal listeriosis]. Zhonghua Nei Ke Za Zhi. 2015;54(9):763-767. Chinese.

58. Hasbún J, Sepúlveda-Martínez A, Haye MT, Astudillo J, ParraCordero M. Chorioamnionitis caused by Listeria monocytogenes: a case report of ultrasound features of fetal infection. Fetal Diagn Ther. 2013;33(4):268-271. doi:10.1159/000345091

59. Bortolussi R, McGregor DD, Kongshavn PA, et al. Host defense mechanisms to perinatal and neonatal Listeria monocytogenes infection. Surv Synth Pathol Res. 1984;3(4):311-332.

60. Gray JW, Barrett JF, Pedler SJ, Lind T. Faecal carriage of listeria during pregnancy. Br J Obstet Gynaecol. 1993;100(9):873-874. doi:10.1111/j.1471-0528.1993.tb14324.x

61. Pezeshkian R, Fernando N, Carne CA, Simanowitz MD. Listeriosis in mother and fetus during the first trimester of pregnancy. Case report. Br J Obstet Gynaecol. 1984;91(1):85-86. doi:10.1111/j.1471-0528.1984.tb05284.x

62. McLauchlin J. Human listeriosis in Britain, 1967-85, a summary of 722 cases. 1. Listeriosis during pregnancy and in the newborn. Epidemiol Infect. 1990;104(2):181-189. doi:10.1017/ S0950268800059343

63. Silk BJ, Date KA, Jackson KA, et al. Invasive listeriosis in the foodborne diseases active surveillance network (FoodNet), 2004-2009: further targeted prevention needed for higher-risk groups. Clin Infect Dis. 2012;54(Suppl 5):S396-404. doi:10.1093/cid/cis268

64. Awofisayo A, Amar C, Ruggles R, et al. Pregnancy-associated listeriosis in England and Wales. Epidemiol Infect. 2015;143 (2):249-256. doi:10.1017/S0950268814000594

65. Williams D, Irvin EA, Chmielewski RA, Frank JF, Smith MA. Dose-response of Listeria monocytogenes after oral exposure in pregnant guinea pigs. J Food Prot. 2007;70(5):1122-1128. doi:10.4315/0362-028X-70.5.1122

66. Torvaldsen S, Kurinczuk JJ, Bower C, Parsons DE, Roberts CL. Listeria awareness among new mothers in Western Australia. Aust N Z J Public Health. 1999;23(4):362-367. doi:10.1111/j.1467842X.1999.tb01276.x

67. Silver HM. Listeriosis during pregnancy. Obstet Gynecol Surv. 1998;53(12):737-740. doi:10.1097/00006254-199812000-00004

68. Benshushan A, Tsafrir A, Arbel R, Rahav G, Ariel I, Rojansky N. Listeria infection during pregnancy: a 10 year experience. Isr Med Assoc J. 2002;4(10):776-780. 
69. Posfay-Barbe KM, Wald ER. Listeriosis. Pediatr Rev. 2004;25 (5):151-159. doi:10.1542/pir.25-5-151

70. Berche P, Reich KA, Bonnichon $\mathrm{M}$, et al. Detection of anti-listeriolysin $\mathrm{O}$ for serodiagnosis of human listeriosis. Lancet. 1990;335(8690):624-627. doi:10.1016/0140-6736(90) 90411-W

71. Pucci L, Massacesi M, Liuzzi G. Clinical management of women with listeriosis risk during pregnancy: a review of national guidelines. Expert Rev Anti Infect Ther. 2018;16(1):13-21. doi:10.1080/14787210.2018.1417837

72. Jamshidi M, Jahromi AS, Davoodian P, Amirian M, Zangeneh M, Jadcareh F. Seropositivity for Listeria monocytogenes in women with spontaneous abortion: a Case-Control Study in Iran. Taiwan $J$ Obstet Gynecol. 2009;48(1):46-48. doi:10.1016/S10284559(09)60034-6

73. Massacesi M, Oliva N, Pucci L, Brandi G, De Santi M. Listeriolysin $\mathrm{O}$ antibodies detection in pregnant women: results from an Italian pilot study. Ann Ig. 2019;31(4):326-332. doi:10.7416/ai.2019.2294

74. Bubonja-Sonje M, Mustac E, Brunn A, Deckert M, Abram M. Listeriosis in pregnancy: Case Report and Retrospective Study J Matern Fetal Neonatal Med. 2013;26(3):321-323. doi:10.3109/ 14767058.2012.733780

75. Barikbin P, Sallmon H, Hüseman D, et al. Clinical, laboratory, and placental findings in perinatal listeriosis. Fetal Pediatr Pathol. 2016;35(5):307-314. doi:10.1080/ 15513815.2016.1179822

76. Mazor M, Froimovich M, Lazer S, Maymon E, Glezerman M. Listeria monocytogenes. The role of transabdominal amniocentesis in febrile patients with preterm labor. Arch Gynecol Obstet. 1992;252(2):109-112. doi:10.1007/BF02389637

77. Craig S, Permezel M, Doyle L, Mildenhall L, Garland S. Perinatal infection with Listeria monocytogenes. Aust $N Z$ J Obstet Gynaecol. 1996;36(3):286-290. doi:10.1111/j.1479-828X.1996. tb02712.x

78. Disson O, Grayo S, Huillet E, et al. Conjugated action of two species-specific invasion proteins for fetoplacental listeriosis. Nature. 2008;455(7216):1114-1118. doi:10.1038/nature07303

79. Heidarzadeh S, Dallal MMS, Pourmand MR, et al. Prevalence, antimicrobial susceptibility, serotyping and virulence genes screening of Listeria monocytogenes strains at a tertiary care hospital in Tehran, Iran. Iran J Microbiol. 2018;10(5):307-313.

80. Shaw GM, Todoroff K, Velie EM, Lammer EJ. Maternal illness, including fever and medication use as risk factors for neural tube defects. Teratology. 1998;57(1):1-7. doi:10.1002/(SICI)10969926(199801)57:1<1::AID-TERA1>3.0.CO;2-6

81. Aselton P, Jick H, Milunsky A, Hunter JR, Stergachis A. Firsttrimester drug use and congenital disorders. Obstet Gynecol. 1985;65(4):451-455.

82. Ito S, Blajchman A, Stephenson M, Eliopoulos C, Koren G. Prospective follow-up of adverse reactions in breast-fed infants exposed to maternal medication. Am J Obstet Gynecol. 1993;168 (5):1393-1399. doi:10.1016/S0002-9378(11)90771-6

83. Temple ME, Nahata MC. Treatment of listeriosis. Ann Pharmacother. 2000;34(5):656-661. doi:10.1345/aph.19315

84. Thønnings S, Knudsen JD, Schønheyder HC, et al. Antibiotic treatment and mortality in patients with Listeria monocytogenes meningitis or bacteraemia. Clin Microbiol Infect. 2016;22 (8):725-730. doi:10.1016/j.cmi.2016.06.006

85. Hernández-Díaz S, Werler MM, Walker AM, Mitchell AA. Neural tube defects in relation to use of folic acid antagonists during pregnancy. Am J Epidemiol. 2001;153(10):961-968. doi:10.1093/aje/153.10.961
86. Lin KJ, Mitchell AA, Yau WP, Louik C, Hernández-Díaz S Safety of macrolides during pregnancy. Am J Obstet Gynecol. 2013;208(3):221.e1-8. doi:10.1016/j.ajog.2012.12.023

87. Romøren M, Lindbæk M, Nordeng H. Pregnancy outcome after gestational exposure to erythromycin - a population-based register study from Norway. $\mathrm{Br} \quad \mathrm{J}$ Clin Pharmacol. 2012;74 (6):1053-1062. doi:10.1111/j.1365-2125.2012.04286.x

88. Welekidan LN, Bahta YW, Teklehaimanot MG, et al. Prevalence and drug resistance pattern of Listeria monocytogenes among pregnant women in Tigray region, Northern Ethiopia: a Cross-Sectional Study. BMC Res Notes. 2019;12(1):538. doi:10.1186/s13104-019-4566-8

89. Pourkaveh B, Ahmadi M, Eslami G, Gachkar L. Factors contributes to spontaneous abortion caused by Listeria monocytogenes, in Tehran, Iran, 2015. Cell Mol Biol (Noisy-Le-Grand). 2016;62 (9):3-10

90. Sosnowski M, Lachtara B, Wieczorek K, Osek J. Antimicrobial resistance and genotypic characteristics of Listeria monocytogenes isolated from food in Poland. Int $J$ Food Microbiol. 2019;289:1-6. doi:10.1016/j.ijfoodmicro.2018.08.029

91. Zhang X, Niu Y, Liu Y, et al. Isolation and characterization of clinical Listeria monocytogenes in Beijing, China, 2014-2016. Front Microbiol. 2019;10:981. doi:10.3389/fmicb.2019.00981

92. Clark DR, Chaturvedi V, Kinder JM, et al. Perinatal Listeria monocytogenes susceptibility despite preconceptual priming and maintenance of pathogen-specific $\mathrm{CD} 8(+) \mathrm{T}$ cells during pregnancy. Cell Mol Immunol. 2014;11(6):595-605. doi:10.1038/cmi.2014.84

93. Calderón-Gonzalez R, Terán-Navarro H, Frande-Cabanes E, et al. Pregnancy vaccination with gold glyco-nanoparticles carrying Listeria monocytogenes peptides protects against listeriosis and brain- and cutaneous-associated morbidities. Nanomaterials (Basel). 2016;6(8):151. doi:10.3390/nano6080151

94. Elinav H, Hershko-Klement A, Solt I, Glikman D, Nir-Paz R. Pregnancy-associated listeriosis: many beliefs, few facts. Lancet Infect Dis. 2015;15(10):1128-1130. doi:10.1016/S1473-3099(15) 00302-3

95. Madjunkov M, Chaudhry S, Ito S. Listeriosis during pregnancy. Arch Gynecol Obstet. 2017;296(2):143-152. doi:10.1007/s00404017-4401-1

96. Imanishi M, Routh JA, Klaber M, et al. Estimating the attack rate of pregnancy-associated listeriosis during a large outbreak. Infect Dis Obstet Gynecol. 2015;2015:201479. doi:10.1155/2015/ 201479

97. Huang YT, Chen SU, Wu MZ, et al. Molecular evidence for vertical transmission of listeriosis, Taiwan. J Med Microbiol. 2006;55(Pt 11):1601-1603. doi:10.1099/jmm.0.46614-0

98. Luo L, Chen X, Payne M, et al. Case report: whole genome sequencing based investigation of maternal-neonatal listeriosis in Sichuan, China. BMC Infect Dis. 2019;19(1):893. doi:10.1186/s12879-019-4551-9

99. Borges SF, Silva JG, Teixeira PC. Survival and biofilm formation of Listeria monocytogenes in simulated vaginal fluid: influence of pH and strain origin. FEMS Immunol Med Microbiol. 2011;62 (3):315-320. doi:10.1111/j.1574-695X.2011.00815.x

100. Sapuan S, Kortsalioudaki C, Anthony M, et al. Neonatal listeriosis in the UK 2004-2014. J Infect. 2017;74(3):236-242. doi:10.1016/j.jinf.2016.11.007 


\section{Publish your work in this journal}

Infection and Drug Resistance is an international, peer-reviewed openaccess journal that focuses on the optimal treatment of infection (bacterial, fungal and viral) and the development and institution of preventive strategies to minimize the development and spread of resistance. The journal is specifically concerned with the epidemiology of

antibiotic resistance and the mechanisms of resistance development and diffusion in both hospitals and the community. The manuscript management system is completely online and includes a very quick and fair peerreview system, which is all easy to use. Visit http://www.dovepress.com/ testimonials.php to read real quotes from published authors. 